\title{
FREE DERIVATION MODULES AND A CRITERION FOR REGULARITY ${ }^{1}$
}

\author{
SELMER MOEN
}

\begin{abstract}
Let $k$ be an algebraically closed field of characteristic zero, $R$ an affine $k$-algebra. We prove that if the ideal of the variety of $R$ can be generated by an $S$-sequence of forms in a polynomial ring $S$, and if the module of $k$-derivations of $R$ into itself is a free $R$ module, then $R$ is regular.
\end{abstract}

1. Introduction. In this paper we investigate the following question: let $R$ be the ring of polynomial functions on an affine variety $V$ over an algebraically closed field $k$ of characteristic zero, $D^{*}(R)$ the module of $k$-derivations of $R$ into itself. Suppose $D^{*}(R)$ is a free $R$-module. Is $R$ then a regular ring? This question was considered by Lipman [2] at the suggestion of Zariski. Lipman proved that $R$ is normal, and gave examples which suggest the truth of an affirmative answer.

The object of this paper is to prove the

THEOREM. Let $R$ be a projective complete intersection, i.e., a residue class ring of a polynomial ring $S=k\left[X_{1}, X_{2}, \cdots, X_{n}\right]$ over $k$ (as above) by an ideal generated by an $S$-sequence of forms. Suppose $D^{*}(R)$ is free. Then $R$ is regular (has finite global dimension).

The proof makes use of the characterization of $D^{*}$ as the module of row-relations on a certain matrix, namely the transpose of the Jacobian matrix associated with the polynomials generating the ideal of the variety $V$. We investigate the connection between the maximal-size minors of this matrix and the matrix obtained from a free basis for $D^{*}$ by writing the elements of that basis as rows. We use these results in a certain Koszul complex related to $D^{*}$ to complete the proof.

The main result of this paper generalizes an example due to Zariski [2, §7], that of a cone in three-space. The author wishes to thank Professor J. Eagon and Professor M. Hochster for many helpful conversations and insights.

Received by the editors November 22, 1971 and, in revised form, October 16, 1972. AMS (MOS) subject classifications (1970). Primary 13H05; Secondary 13B10, 14 M10. Key words and phrases. Regular ring, derivation module, affine algebra, singular prime, Jacobian ideal, projective complete intersection.

${ }^{1}$ This research was supported in part by NSF grant GP-8496.

(c) American Mathematical Society 1973 
2. Notation and preliminaries. Let $k$ be an algebraically closed field of characteristic zero, $S=k\left[X_{1}, X_{2}, \cdots, X_{n}\right], I$ a radical ideal in $S$ generated by an $S$-sequence of forms, $I=\left(F_{1}, F_{2}, \cdots, F_{r}\right)$. Let $R=S / I$; we denote reduction modulo $I$ by lower case letters. Let $J=\left(f_{i j}\right)$ be the Jacobian matrix (with entries in $R$ ) associated to $I$; thus

$$
f_{i j}=\partial F_{i} / \partial X_{j} \bmod I \text {. }
$$

We identify $d$ in $D^{*}(R)$ with the element $\left(d x_{1}, d x_{2}, \cdots, d x_{n}\right)$ of $R^{n}$. Combining this identification with the chain rule, we note that $D^{*}(R)$ may be viewed as the module of row relations on the matrix $J^{t}$. If $u_{1}$, $u_{2}, \cdots, u_{p}$ generate $D^{*}(R)$, with $u_{j}=\left(u_{j 1}, \cdots, u_{j n}\right), 1 \leqq j \leqq p$, set $U=\left(u_{i j}\right)$. We then have

\section{Proposition 1.1. $\quad U J^{t}=0$.}

We assume from now on that $D^{*}(R)$ is free on generators $u_{1}, u_{2}, \cdots, u_{p}$, and note $[2, \S 2]$ that $p+r=n$.

There is an Euler-Poincaré mapping $G$ which associates to each torsion module over $R$ with finite free resolution a principal ideal of $R$ (cf. [3]). Briefly, $G$ is defined as follows. If $M$ is a torsion module of homological dimension (dh) zero, $G(M)=(1)$. If $\operatorname{dh}(M)=1, M=\operatorname{coker} f: R^{n} \rightarrow R^{m}$, then $G(M)=(\operatorname{det}(f))$. If $\infty>\operatorname{dh}(M)>1$, resolve $M$ by torsion modules $T_{j}$ of homological dimension $\leqq 1,0 \rightarrow T_{h} \rightarrow T_{h-1} \rightarrow \cdots \rightarrow T_{2} \rightarrow T_{1} \rightarrow M \rightarrow 0$, and set $G(M)=G\left(T_{1}\right) G\left(T_{2}\right)^{-1} G\left(T_{3}\right) \cdots G\left(T_{h}\right)^{(-1)^{h+1}}$. By localizing at a rank one prime [3], one shows that $G$ is well-defined, and is actually a principal ideal of $R$.

Let $\sigma=\left(\sigma_{1}, \sigma_{2}, \cdots, \sigma_{p}\right), 1 \leqq \sigma_{1}<\sigma_{2}<\sigma_{3} \cdots<\sigma_{p} \leqq n$, be an increasing sequence of $p$ integers between 1 and $n$. Denote by $\Delta_{\sigma}$ the $p \times p$ minor of the $p \times n$ matrix $U$ corresponding to columns $\sigma_{1}, \sigma_{2}, \cdots, \sigma_{p}$, by $\tau_{\sigma}$ the $r \times r$ minor of $J^{t}$ obtained by deleting rows $\sigma_{1}, \sigma_{2}, \cdots, \sigma_{p}$.

Proposition 1.2. There is a unit $\mu \in R$ such that, for any $\sigma, \tau_{\sigma}=$ $(-1)^{|\sigma|} \mu \Delta_{\sigma}$, where $|\sigma|=\sum_{i=1}^{p} \sigma_{i}$.

Proof. We show first that $\left(\tau_{\sigma}\right)=\left(\Delta_{\sigma}\right)$ by using the Euler-Poincaré (multiplicativity on exact sequences) property of the mapping $G$ defined above. The result stems from an idea of Kramer [1].

Suppose for simplicity that $\sigma=(1,2, \cdots, p)$. There are two cases:

Case 1. $\tau_{\sigma} \neq 0$. Let $\rho_{i}$ denote the $i$ th row of the matrix $J^{t}$, and let $P$ be the submodule of $R^{r}$ generated by the $\rho_{i}$. Let $M$ be the submodule of $P$ generated by $\rho_{p+1}, \rho_{p+2}, \cdots, \rho_{n}$. We obtain an exact sequence $0 \rightarrow K \rightarrow$ $R^{n} \rightarrow P / M \rightarrow 0$ by factoring the canonical map $R^{n} \rightarrow P \rightarrow 0, e_{i} \rightarrow \rho_{i}$, where $e_{i}$ are the usual basis elements of $R^{n}$. We claim $K=D^{*} \oplus e_{p+1} \oplus e_{p+2} \oplus \cdots \oplus e_{n}$. 
For

$$
\begin{aligned}
K & =\left\{\sum_{i=1}^{n} a_{i} e_{i} \in R^{n}: \sum_{i=1}^{n} a_{i} \rho_{i} \in M\right\} \\
& =\left\{\sum_{i=1}^{n} a_{i} e_{i}: \text { there are } b_{p+1}, \cdots, b_{n} \text { in } R\right. \\
& \text { with } \left.\sum_{i=1}^{p} a_{i} \rho_{i}+\sum_{i=p+1}^{n}\left(a_{i}-b_{i}\right) \rho_{i}=0\right\} \\
& =D^{*} \oplus e_{p+1} \oplus e_{p+2} \oplus \cdots \oplus e_{n} .
\end{aligned}
$$

The proof of the last equality follows. Let $\sum_{i=1}^{n} a_{i} e_{i} \in K$. Then there are $b_{i}$ in $R$ such that

hence

$$
d=\sum_{i=1}^{p} a_{i} e_{i}+\sum_{i=p+1}^{n}\left(a_{i}-b_{i}\right) e_{i} \in D^{*}
$$

$$
\sum_{i=1}^{n} a_{i} e_{i} \in D^{*} \oplus e_{p+1} \oplus \cdots \oplus e_{n} .
$$

But if $\sum_{i=1}^{n} a_{i} e_{i}$ is an element of $D^{*} \oplus e_{p+1} \oplus e_{p+2} \oplus \cdots \oplus e_{n}$, say

$$
\sum_{i=1}^{n} a_{i} e_{i}=\sum_{i=1}^{p} r_{i} u_{i}+\sum_{i=p+1}^{n} b_{i} e_{i},
$$

then $\sum_{i=1}^{p} a_{i} e_{i}+\sum_{i=p+1}^{n}\left(a_{i}-b_{i}\right) e_{i} \in D^{*}$, completing the proof of the equality.

The sum is direct: suppose $\sum_{i=p+1}^{n} r_{i} e_{i} \in D^{*}$. Then $\sum_{i=p+1}^{n} r_{i} \rho_{i}=0$, so $\left(r_{p+1}, r_{p+2}, \cdots, r_{n}\right)$ is a solution of the $r \times r$ linear homogeneous system of equations with coefficient matrix whose columns are the $\rho_{i}$. The determinant of this matrix, $\tau_{\sigma}$, is not zero; hence $r_{p+1}=r_{p+2}=\cdots=r_{n}=0$.

Thus $K$ is free of rank $n$, and $G(P / M)=\left(\Delta_{\sigma}\right)$. Now from the exact sequence $0 \rightarrow P / M \rightarrow R^{r} / M \rightarrow R^{r} / P \rightarrow 0$, we get

$$
\left(\tau_{\sigma}\right)=G\left(R^{r} / M\right)=G\left(R^{r} / P\right) G(P / M)=(\lambda)\left(\Delta_{\sigma}\right),
$$

where $\lambda$ is a unit in $R$. To see that $\lambda$ is a unit in $R$, suppose it were not. $\lambda$ is not a zero divisor, so suppose $q$ is a grade one, rank one prime containing it. Since $R$ is normal $[2, \S 3]$, the singular locus of $R$ has grade $\geqq 2$. But the singular primes of $R$ are also the primes containing the maximalsize minors of $J$, which are contained in $\operatorname{Ann}\left(R^{r} / P\right)$. Hence $\left(R^{r} / P\right)_{q}=0$, implying $(\lambda)_{q}=G\left(R^{r} / P\right)_{q}=G\left(\left(R^{r} / P\right)_{q}\right)=(1)$, contradiction. This completes Case 1.

Case 2. $\tau_{\sigma}=0$. Then there is a nontrivial solution to the $r \times r$ system of equations determined by the $\rho_{i}$ as before, say $\sum_{i=p+1}^{n} r_{i} \rho_{i}=0$. Then 
$\sum_{i=p+1}^{n} r_{i} e_{i} \in D^{*}$, so there are $t_{i}$ in $R$ not all zero such that $\sum_{i=p+1}^{n} r_{i} e_{i}=$ $\sum_{j=1}^{p} t_{j} u_{j}$. But this means that the $t_{j}$ give a nontrivial solution to the $p \times p$ system of equations determined by the first $p$ columns of $U$; the determinant of this matrix is $\Delta_{\sigma}$, hence $\Delta_{\sigma}=0$, completing Case 2 .

We have now shown that, for any $\sigma$, there is a unit $\mu_{\sigma}$ in $R$ such that $\tau_{\sigma}=\mu_{\sigma} \Delta_{\sigma}$. To see that $\mu=\mu_{\sigma}$ is independent of $\sigma$, we use an argument from linear algebra. Consider the $n \times n$ matrix formed by writing the rows of $J$ beneath the rows of $U$. Let $K$ denote the quotient field of $R$; by tensoring with $K$ we may assume that the entries of this matrix are in $K$.

Thus we have an $n \times n$ matrix over $K$ whose last $r$ rows are orthogonal to the first $p$ rows. We have the

Lemma. Let $A=\left(a_{i j}\right)$ be an $n \times n$ matrix with entries in a field $K$. Denote the ith row of $A$ by $A_{i}$, and assume that $A_{i} A_{j}^{t}=0$ for $1 \leqq i \leqq s$, $s+1 \leqq j \leqq n$. Further, assume that $A_{1}, A_{2}, \cdots, A_{s}$ and $A_{s+1}, A_{s+2}, \cdots, A_{n}$ are linearly independent over $K$. Then there is an element $\mu$ of $K$ such that $\tau_{\sigma}=(-1)^{|\sigma|} \mu \Delta_{\sigma}$ for any choice of $s$ indices as before, where $\Delta_{\sigma}$ is the $s \times s$ minor corresponding to $\sigma$ from the first s rows of $A$, and $\tau_{\sigma}$ the corresponding $r \times r$ minor from the last $r=n-s$ rows of $A$.

To prove the lemma, assume first that

$$
A=\left(\begin{array}{cc}
I_{s} & M \\
N & I_{r}
\end{array}\right),
$$

where $I_{s}\left(\right.$ resp. $I_{r}$ ) is the $s \times s$ (resp. $\left.r \times r\right)$ identity matrix, so that $N=-M^{t}$. Fix $\sigma$ and assume $\sigma_{k} \leqq s<\sigma_{k+1}$. Then

$$
\Delta_{\sigma}=(-1)^{\Sigma_{i-1}^{k} \sigma_{i}-\left(k^{2}-3 k\right) / 2} \Delta_{\sigma}^{*},
$$

where $\Delta_{\sigma}^{*}$ is an $(s-k) \times(s-k)$ minor of $M$.

If $\xi$ denotes the set of $r$ indices complementary to $\sigma$, we have $\tau_{\sigma}=$ $(-1)^{m} \Delta_{\sigma}^{*}$, where $m$ is given by the formula

$$
\begin{aligned}
m= & (s-k)(r-s+k) \\
& +\sum_{i=1}^{r-s+k} \xi_{s-k+i}-\frac{s(r-s+k)^{2}-3(r-s+k)}{2}+(s-k),
\end{aligned}
$$

using the fact that $N=-M^{t}$.

Hence $\tau_{\sigma}= \pm(\operatorname{sgn} \sigma) \Delta_{\sigma}$, where the first sign is independent of $\sigma$, as follows by checking each term of $m$ and observing that those terms which depend upon $\sigma$ do so exactly as $\operatorname{sgn} \sigma=(-1)^{|\sigma|}$.

If $A$ is now any $n \times n$ matrix satisfying the hypotheses of the lemma, 
assume (as we may) that for $\sigma(0)=(1,2, \cdots, s), \Delta_{\sigma(0)} \neq 0, \tau_{\sigma(0)} \neq 0$. Write

$$
A=\left(\begin{array}{cc}
A_{11} & A_{12} \\
A_{21} & A_{22}
\end{array}\right)
$$

where $A_{11}$ is the $s \times s$ matrix whose determinant is $\Delta_{\sigma(0)}, A_{22}$ the $r \times r$ matrix whose determinant is $\tau_{\sigma(0)}$. Then the matrix

$$
A^{\prime}=\left(\begin{array}{cc}
A_{11}^{-1} & 0 \\
0 & A_{22}^{-1}
\end{array}\right)\left(\begin{array}{ll}
A_{11} & A_{12} \\
A_{21} & A_{22}
\end{array}\right)=\left(\begin{array}{cc}
I_{s} & A_{11}^{-1} A_{12} \\
A_{22}^{-1} A_{21} & I_{r}
\end{array}\right)
$$

satisfies the hypotheses of the lemma, hence its conclusion. It is immediate that the $s \times s$ minors satisfy $\Delta_{\sigma}^{\prime}=\left(\Delta_{\sigma(0)}\right)^{-1} \Delta_{\sigma}$, and that the $r \times r$ minors satisfy $\tau_{\sigma}^{\prime}=\left(\tau_{\sigma(0)}\right)^{-1} \tau_{\sigma}$.

Combining these results, we obtain

$$
\begin{aligned}
\tau_{\sigma} & =\tau_{\sigma(0)} \tau_{\sigma}^{\prime}=\tau_{\sigma(0)}\left[ \pm(\operatorname{sgn} \sigma) \Delta_{\sigma}^{\prime}\right] \\
& =\tau_{\sigma(0)}\left[ \pm(\operatorname{sgn} \sigma)\left(\Delta_{\sigma(0)}\right)^{-1}\right]=\left(\tau_{\sigma(0)} / \Delta_{\sigma(0)}\right)( \pm \operatorname{sgn} \sigma) \Delta_{\sigma} .
\end{aligned}
$$

This completes the proof of the lemma.

To finish the proof of the proposition, we need only remark that our previous argument shows that $\tau_{\sigma(0)} / \Delta_{\sigma(0)}$ is a unit in $R$, not merely in the quotient field of $R$. Q.E.D.

3. Main result. Before stating the theorem and completing its proof, we remark that the property of homogeneity implies (Euler's formula) that $x=\left(x_{1}, x_{2}, \cdots, x_{n}\right)$ is an element of $D^{*}(R)$, where $x_{i}=$ image of $X_{i}$ in $R=S / I$.

THEOREM. Let $R=S / I$ be a reduced projective complete intersection. Assume that $D^{*}(R)$ is free. Then $R$ is regular (has finite global dimension). (Note: it follows that I must be generated by forms of degree one.)

Proof. Suppose $R$ is not regular. We may then assume that $x$ is a member of a free basis for $D^{*}(R)$ (cf. Seidenberg [4]). We apply Proposition 1.2 to obtain a contradiction.

Let $\wedge S^{n}$ be the exterior algebra on $S^{n}$, where $S^{n}=S V_{1}+S V_{2}+\cdots+$ $S V_{n}$ with $V_{i}$ the usual basis for $S^{n}$. Define maps $d_{i}: \bigwedge S^{n} \rightarrow \wedge S^{n}$ by

$$
d_{i} V_{j}=F_{i j} / m_{i} \quad\left(m_{i}=\operatorname{deg} F_{i}\right)
$$

and require that each $d_{i}$ be a homogeneous derivation of degree $(-1)$ on $S^{n}$ (i.e., $\left.d_{i}(p \wedge q)=d_{i}(p) \wedge q+(-1)^{\operatorname{deg} q} p \wedge d_{i}(q)\right)$. Finally, let $V=V_{1} \wedge V_{2} \wedge \cdots \wedge$ $V_{n}$ generate $\wedge^{n} S^{n}$, and set $D_{k}=d_{1} \circ d_{2} \circ \cdots \circ d_{k}, 1 \leqq k \leqq r$. We shall show that the assumption on $x$ implies that $V \in M\left(\bigwedge^{n} S^{n}\right)$, where $M$ is 
the ideal generated by $X_{1}, X_{2}, \cdots, X_{n}$ in $S$, contradicting the definition of $V$.

Fix a free basis $x, u_{2}, u_{3}, \cdots, u_{p}$ of $D^{*}(R)$. Let $X$ denote the element $\left(X_{1}, X_{2}, \cdots, X_{n}\right)$ of $S^{n}$. The quotient map $S \rightarrow R$ induces a homomorphism $S^{n} \rightarrow R^{n}$ which maps $X$ on $x$. For $2 \leqq i \leqq p$, let $U_{i}$ be an element of $S^{n}$ which is mapped on $u_{i}$ by this induced homomorphism.

We also have an induced homomorphism of exterior algebras; let $\left[D_{r} V\right]$ denote the image under this homomorphism of $D_{r} V$ in $\wedge^{p} R^{n}$. Then the conclusion of Proposition 1.2 can be stated

$$
x \wedge u_{2} \wedge u_{3} \wedge \cdots \wedge u_{p}=\left[D_{r} V\right] .
$$

This equality in $\wedge R^{n}$ can be rewritten in $\wedge S^{n}$ as $X \wedge U_{2} \wedge U_{3} \wedge \cdots \wedge U_{p}=$ $D_{r} V-\sum_{i=1}^{r} F_{i} E_{i}^{p}$, where $E_{i}^{p} \in \bigwedge^{p} S^{n}, 1 \leqq i \leqq r$.

Since $X \wedge V=0$, we have

$$
\begin{aligned}
0= & D_{r}(X \wedge V)=d_{1} \circ d_{2} \circ \cdots \circ d_{r}(X \wedge V) \\
= & d_{1} \circ d_{2} \circ \cdots \circ d_{r-1}\left(d_{r}(X) \wedge V \pm X \wedge d_{r}(V)\right) \\
= & d_{1} \circ d_{2} \circ \cdots \circ d_{r-1}\left(F_{r} V \pm X \wedge d_{r}(V)\right) \\
= & \cdots=F_{r} D_{r-1}(V) \\
& \pm \operatorname{terms} \operatorname{in}\left(F_{1}, F_{2}, \cdots, F_{r-1}\right) \wedge S^{n} \pm X \wedge D_{r}(V) .
\end{aligned}
$$

But

$$
\begin{aligned}
X \wedge D_{r}(V) & =X \wedge\left(X \wedge U_{2} \wedge \cdots \wedge U_{p}\right)-\sum_{i=1}^{r} F_{i}\left(X \wedge E_{i}^{p}\right) \\
& =-\sum_{i=1}^{r} F_{i}\left(X \wedge E_{i}^{p}\right) .
\end{aligned}
$$

Combining yields $F_{r}\left(D_{r-1}(V) \pm X \wedge E_{r}^{p}\right) \in\left(F_{1}, F_{2}, \cdots, F_{r-1}\right) \wedge S^{n}$. But $F_{1}, F_{2}, \cdots, F_{r}$ is an $S$-sequence on $\Lambda S^{n}$, hence $D_{r-1}(V) \pm\left(X \wedge E_{r}^{p}\right)=$ $\sum_{i=1}^{r-1} F_{i} E_{i}^{p+1}$, where $E_{i}^{p+1} \in \bigwedge^{p+1} S^{n}, 1 \leqq i \leqq r-1$.

Repeat the argument:

$$
\begin{aligned}
0 & =D_{r-1}(X \wedge V)=D_{r-2}\left(F_{r-1} V \pm X \wedge D_{r-1}(V)\right) \\
& =\cdots=F_{r-1} D_{r-2}(V)+\operatorname{terms} \text { in }\left(F_{1}, \cdots, F_{r-2}\right) \wedge S^{n} \pm X \wedge D_{r-1}(V) \\
& =F_{r-1}\left(D_{r-2}(V) \pm X \wedge E_{r-1}^{p+1}\right)+\operatorname{terms} \text { in }\left(F_{1}, \cdots, F_{r-2}\right) \wedge S^{n} .
\end{aligned}
$$

Hence $D_{r-2}(V) \pm X \wedge E_{r-1}^{p+1}=\sum_{i=1}^{r-2} F_{i} E_{i}^{p+2}$.

Repeating the argument $r-1$ times, we obtain $D_{1}(V) \pm X \wedge E_{2}^{n-2}=F_{1} E_{1}^{n-1}$. Then

$$
\begin{aligned}
0 & =D_{1}(X \wedge V)=d_{1}(X \wedge V) \\
& =F_{1} V \pm X \wedge d_{1}(V)=F_{1}\left(V-X \wedge E_{1}^{n-1}\right) .
\end{aligned}
$$

Hence $V=X \wedge E_{1}^{n-1} \in M\left(\bigwedge^{n} S^{n}\right)$, contradicting the choice of $V$. Q.E.D. 


\section{REFERENCES}

1. H. Kramer, Eine Bemerkung zu einer Vermutung von Lipman, Ark. Mat. 20 (1969), 30-35.

2. J. Lipman, Free derivation modules on algebraic varieties, Amer. J. Math. 87 (1965), 874-898. MR 32 \#4130.

3. R. E. MacRae, On an application of the Fitting invariants, J. Algebra 2 (1965), 153-169. MR 31 \#2296.

4. A. Seidenberg, Differential ideals in rings of finitely generated type, Amer. J. Math. 89 (1967), 22-42. MR 35 \#2902.

Department of Mathematics, University of Minnesota, Minneapolis, MinneSOTA 55455

Current address: Department of Mathematics, Mount Union College, Alliance, Ohio 44601 